\title{
INFRACLAVICULAR BRACHIAL PLEXUS BLOCK FOR ACUTE COMPARTMENT SYNDROME
}

\author{
YENIOCAK T, CANBOLAT N, \\ Baltalimani Metin Sabanci Bone and Joint Diseases Education and Research Hospital \\ İstanbul, TURKEY \\ Department of Anaesthesiology
}

\begin{abstract}
Background and Aims:
Acute compartment syndrome (ACS) is an orthopedic emergency. Increased fascial compartment pressure can compromise circulation and function of tissues, may lead to irreversible neuromuscular ischemic damage in four to six hours after the symptoms emerge. The aim of the study is to report our experience in eight ACS cases.
\end{abstract}

\section{Methods:}

With the approval of the ethics committee; we observed eight ACS cases that were performed with ultrasound guided infraclavicular brachial plexus block (USGICB) for fasciotomy procedure with diluted local anesthetic (LA) dose.

Case 1: A 65 year old male with distal radius fracture

Case 2: A 37 year old male with deep tissue enfection on forearm

Case 3: A 70 year old male with distal radius and ulna fracture Case 4: A 25 year old male with deep tissue enfeciton on forearm .

Case 5: A 17 year old male with multiple metacarp fractures

Case 6: A 28 year old male with multiple metacarp fractures

Case 7: A 76 year old male with crush injury on wrist , 36 hours delayed admission to emergency department

Case 8: A 56 year old male with multiple metacarp fractures

\section{Results:}

Eight patients were admitted to emergency department with severe pain and pallor on forearm .All patients have the same story ; first administration of hospital after injuries and after occuring severe pain. None of them has surgery. They planned for faciotomy procedure immediately. Following electrocardiography, peripheral $\mathrm{O}_{2}$ saturation $\left(\mathrm{SPO}_{2}\right)$ and non invasive blood monitorisation, intravenous acces were established on the non operative upper limb, patients were placed in supine position , heads were turned to the opposite side of the blocks would be performed. The skin was aseptically prepared by poviodine iodine and single shot USGICB was performed with a mixture of $10 \mathrm{ml}$ of $\% 0.25$ bupivacaine and $10 \mathrm{ml}$ of $\% 1$ lidocaine. Fasciotomy was performed without any complications. Perioperatively no other analgesia was administered. The patients needed the first analgesic approximately four hours after surgery.Seven patients recovered without any sequellae, however, the patient with the crush injury died because of multi organ dysfunction due delayed admission to the hospital.

\section{Conclusion:}

USGICB is an effective method for postoperative pain management. Whether analgesia provided by USGICB technique would mask the symptom of ACS and consequently delay the diagnosis of ACS remains a controversial issue. USGICB with low dose LA can be comfortable and effective for postoperative pain management in already diagnosed ACSs and post fasciotomy period. ACS is very painful and therefore relieving pain and ensuring patient's comfort is very important in this process. Diluted doses of LA can be applied to prevent masking ACS 